\title{
IQ imbalance reduction in a SMI multi-antenna receiver by using a code multiplexing front-end
}

\author{
Matthieu Gautier ${ }^{1,2}$, Pierre-François Morlat ${ }^{1}$ and Guillaume Villemaud ${ }^{1}$ \\ ${ }^{1}$ Université de Lyon, INRIA, INSA-Lyon, CITI - F-69621 - France \\ ${ }^{2}$ Orange Labs - 28, chemin du vieux chêne - 38243 Meylan - France
}

\begin{abstract}
In this paper, we address the IQ imbalance sensibility of the code multiplexing front-end architecture. This innovative architecture has been recently proposed in order to reduce the analog complexity of an antenna diversity receiver front-end. An interesting characteristic of this structure is that the resulting IQ imbalance is equal for each received baseband signal. Associated with Single Matrix Inversion algorithm, this property ensures a high IQ imbalance robustness. A global antenna diversity system including analog front-end and digital processing has been implemented in order to perform simulation validation. Results show that the bit error rate does not increase significantly with the multiplexing and this increase is compensated for a high IQ imbalance.
\end{abstract}

\section{INTRODUCTION}

Introduced in 1984 by J.H. Winters [1], the antenna diversity techniques are an interesting solution for advanced communication systems. They are used to increase the capacity and performances (quality of service QoS, datarate, ...) of wireless networks. Several algorithms exist to take advantage of antenna diversity and to combine different signals arriving at the same receiver [6]. In the 802.11g standard [2] study case, receivers are mostly used in indoor environments, with large angular spread, so it is very difficult to use high complexity algorithms such as ESPRIT or MUSIC [3] in order to compute the direction of arrival of signal of interest. Therefore, we prefer to take advantage of the training sequence used in $802.11 \mathrm{~g}$ standard and to compute the optimal combination of different signals with the Sample Matrix Inversion (SMI) [4]. This technique is efficient to increase capacity and performance, as well as to reduce the sensibility to IQ imbalance [5]. However, this compensation is effective when the IQ imbalance is the same on each chain of the receiver, which is not really a realistic assumption.

Recently, a new architecture of multi-antenna front-end architecture has been proposed in order to reduce the analog complexity. This architecture is based on code multiplexing and has been introduced by authors in [6]. A global study has been done including Bit Error Rate (BER) performances, power consumption evaluation and implementation considerations. Some results already submitted for publication are given in this paper but here the main purpose is to assess the IQ imbalance sensibility of this type of receiver. We compare its performances to the classical structure for realistic IQ imbalance, the two systems using SMI processing. In order to evaluate this new antenna diversity receiver, BER simulations have been performed.
This paper consists of 5 parts. Following this introduction, Section II describes the multi-antenna receiver and the new antenna diversity front-end structure is presented. In Section III, the theoretical models of this structure are given, including IQ imbalance. Section IV details some simulated performances. Finally, conclusions are drawn and follow-ups are provided.

\section{Multi-Antenna ReCEIVER}

\section{A. Classical architecture}

In order to have a performance improvement, a transceiver using antenna diversity [1] has to use several antennas for its transmitter and/or receiver. Fig. 1 shows the structure of an antenna diversity transceiver in the SIMO (Single-Input Multiple-Output) configuration. The multi-antenna receiver is composed of two parts: an analog one that transforms the radiofrequency (RF) signal $r_{k}(t)$ in baseband signals in-phase $I_{k}(t)$ and in-quadrature $Q_{k}(t)$ [7] and a digital one that performs the antenna diversity processing.

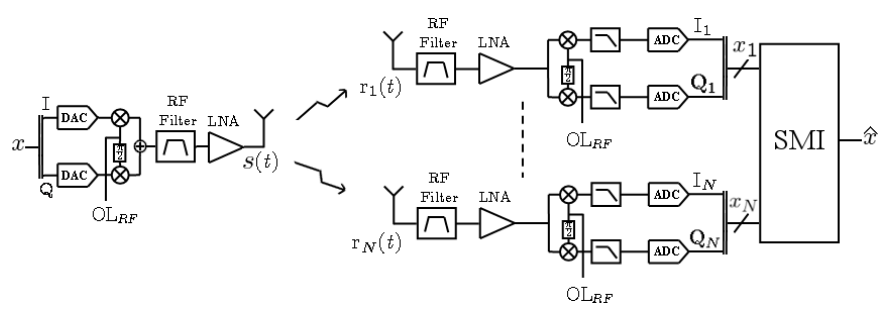

Fig. 1. Classical architecture of an antenna diversity receiver.

The classical analog front-end associated to antenna diversity receivers uses one dedicated analog chain for each of the $N$ antennas. The receiver of the Fig. 1 uses a homodyne architecture [8]. Each chain is composed of a SAW RF filter (Surface Acoustic Wave filter) for the band selection, an LNA (Low Noise Amplifier) and an IQ demodulator that recovers the in-phase and in-quadrature baseband signals. The $I_{k}(t)$ and $Q_{k}(t)$ signals of the $k^{t h}$ antenna are then digitized by two analog to digital converters (ADC). The baseband signals $x_{k}(t)=I_{k}(t)+j Q_{k}(t)$ are processed by the SMI algorithm in order to compute $\hat{x}(t)$.

The antenna diversity digital processing used in this study is the classical SMI [4]. It uses the training sequence of $802.11 \mathrm{~g}$ standard [8] in order to compute the optimal combination 
(Fig. 2) of different signals using an adaptive algorithm based on a Minimum Mean Square Error (MMSE) criterion. A simple adaptive algorithm is the Least Mean Square (LMS) which has a low complexity of $O(N)$. But this algorithm has a very slow convergence. Taking into account that the training sequence time of an $802.11 \mathrm{~g}$ burst is short, it is preferable to use SMI which offers a faster convergence, even if SMI has a more important complexity of $O\left(N^{3}\right)$.

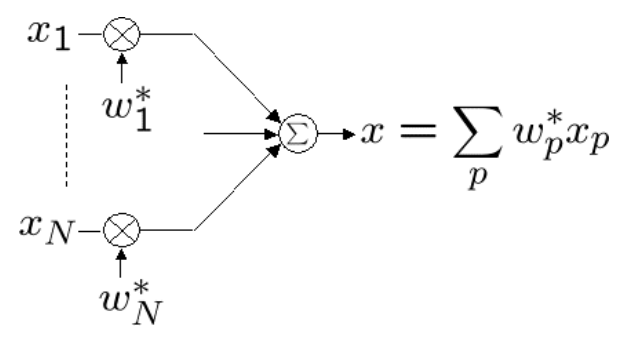

Fig. 2. The multi-antenna adaptive processing.

We apply the SMI algorithm in the temporal domain, directly after sampling. We define an input signal vector by $X(k)=$ $\left[x_{1}(k), \ldots, x_{N}(k)\right]^{T}$ where []$^{T}$ denotes the transpose operation. The covariance matrix $R$ and the cross covariance vector $r$ are estimated with $K$ samples of the signal vector ( $K$ is the number of sample of the training sequence $u$ ):

$$
R=\frac{1}{K} \sum_{k=1}^{K} X^{H}(k) X(k),
$$

where ${ }^{H}$ denotes the transpose and conjugate operation.

$$
r=\frac{1}{K} \sum_{k=1}^{K} X^{H}(k) u(k) .
$$

Denoting $w=\left[w_{1}, \ldots, w_{N}\right]^{T}$ the complex weights to applied to different signals, an approximate solution to the MMSE problem is computed as:

$$
w=R^{-1} r .
$$

\section{B. The code multiplexing architecture}

The RF stack-up architecture described in Fig. 1 is an obvious choice: having $N$ separated dedicated chains allows the demodulation of each branch with a significant quality (high Signal to Noise Ratio - SNR at the ADC input). However, this choice enforces a high complexity of the analog frontend. The aim of the structure shown in Fig. 3 is to reduce this complexity without decreasing the SNR quality after branches demodulation.

The idea was to explore the use of a single common frontend for the processing of signals received by the $N$ antennas. The use of one common analog chain underlies the idea of multiplexing the different branches on a single frontend. The code domain appears to be the most suited for an antenna diversity receiver. In order to achieve the time and frequency overlapping between each antenna contribution, decorrelation can be done by the Direct Sequence Spread
Spectrum technique (DSSS). This technique is the basis of CDMA multiple access technology [9]. The spread spectrum allows a multiplexing which is neither time nor frequency, but a code multiplexing.

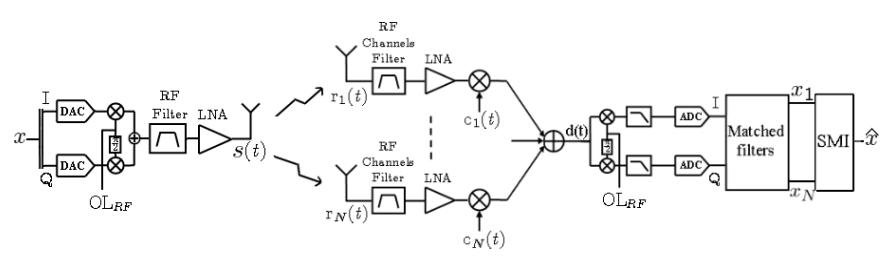

Fig. 3. Code multiplexing architecture of an antenna diversity receiver.

The DSSS technique consists in allocating an orthogonal spreading code to each branch. The signals $r_{k}(t)$ are multiplied by the periodical codes $c_{k}(t)$ which are pseudorandom sequences of $N$ binary entities having a rate $N$ times higher than the symbol. Thus, the resulting bandwidth of the signal spectrum is $N$ times larger than those of the received signal $r_{k}(t)$. The sum of $N$ encoded signals is then performed in order to generate the radio-frequency multiplex signal $d(t)$. This signal is then transformed by an IQ demodulator. After the sampling step, the decoding of each contribution is performed, it consists in matched filters composed of digital filters followed by subsampling operations.

The theoretical aspects of the proposed structure are given in the next section.

This architecture works for any multi-antenna schemes: for every antenna array and every digital algorithm. However, a limitation of the concept of using orthogonal spreading codes is that optimally only an even number $(2,4,8, \ldots)$ of antennas can be received.

The synchronization between coding and decoding is not such an important issue as during an UMTS transmission. Indeed, the propagation delay of the spread signal through the circuit path is well-predicted through accurate circuit analysis and simulation. Therefore, attaining synchronization between the spreading and despreading codes is a trivial matter. For this reason, time delay between the spreading and despreading codes is neglected in this study.

The SNR study, already submitted for publication, has shows that the BER does not increase so much with the multiplexing, this degradation is due to digital aliasing during the subsampling operation. Future work is to reduce this aliasing effect. A complexity study shows that the proposed structure reduces significantly the power consumption of the antenna diversity front-end. This structure outperforms the classical structure for a number of antennas above 2 . A consumption reduction of $25 \%$ is reached for $N=8$ antennas.

The IQ imbalance sensitivity of this structure has not been assessed yet and is the main subject of this paper. Theoretical considerations are given in the next section. 


\section{IQ IMBALANCE IN THE CODE MULTIPLEXING ARCHITECTURE}

In this section, the system model of the code multiplexing architecture is presented, including IQ imbalance. The novel antenna diversity receiver is depicted in Fig. 3 and consists in 2 parts: the analog multiplexing and the digital demultiplexing.

\section{A. System model and IQ imbalance}

Analog coding: The digital transmitted message is made of complex symbols $x[i]$ which depend on the digital modulation (BPSK, QPSK, QAM, OFDM, ... ). These symbols are transmitted at the symbol rate $D_{s}=1 / T_{s}$ where $T_{s}$ is the symbol duration. The digital baseband signal (analog signal carrying digital information), with a rectangular pulse shaping, is written:

$x(t)=\sum_{i=-\infty}^{+\infty} x[i] p_{T_{s}}\left(t-i T_{s}\right)$ with $p_{T_{s}}=\left\{\begin{array}{l}1 \text { if } 0 \leq t<T_{s}, \\ 0 \text { else. }\end{array}\right.$

The RF transmitted signal is real and is defined by:

$$
s(t)=x(t) e^{j 2 \pi f_{0} t}+x^{*}(t) e^{-j 2 \pi f_{0} t},
$$

with $f_{0}$ the RF modulating frequency.

In an ideal transmission, the received signal on the $k^{t h}$ antenna is expressed by:

$$
r_{k}(t)=x_{k}(t) e^{j 2 \pi f_{0} t}+x_{k}^{*}(t) e^{-j 2 \pi f_{0} t},
$$

with $x_{k}(t)$ the baseband signal received on the $k^{t h}$ antenna. In order to perform the code multiplexing of all the branches, each received signal is spread using a code $c_{k}(t), k=1, \ldots N$. The pseudo-random sequences are $T_{s}$-periodic. By using a limited symbol duration code, repeated indefinitely, the system has an easier sequence generation as well as a better despreading synchronization. For a given branch $k$, a set of $N$ binary (complex binary) chips $\left\{c_{k}[n], n=0, \ldots N-1\right\}$ is used. This sequence is called "spreading code". The periodic code $c_{k}^{p e r}(t)$ is the periodic pseudo-random sequence:

$$
c_{k}^{p e r}(t)=\sum_{i=-\infty}^{+\infty} c_{k}(t) p_{T_{s}}\left(t-i T_{s}\right) .
$$

The non-periodic code is defined by:

$$
c_{k}(t)=\sum_{n=0}^{N-1} c_{k}[n] p_{T_{c}}\left(t-n T_{c}\right),
$$

where $T_{c}$ is the chip duration and $N=\frac{T_{s}}{T_{c}}$ is the code length. The use of codes with a $N$ length allows the reception of $N$ antennas.

The $k^{t h}$ antenna contribution after spreading $d_{k}(t)$ is expressed by:

$$
d_{k}(t)=c_{k}^{p e r}(t) r_{k}(t)
$$

By replacing $r_{k}(t)$ by (6), we get:

$$
d_{k}(t)=c_{k}^{p e r}(t)\left\{x_{k}(t) e^{j 2 \pi f_{0} t}+x_{k}^{*}(t) e^{-j 2 \pi f_{0} t}\right\} .
$$

Once the coding operation ended for each antenna, the new signals intercorrelations depend only on the codes intercorrelations. So, signals can be clearly overlapped in time and frequency, as they are separable by their spreading sequence. The adding operation between the spread contributions can be done:

$$
d(t)=\sum_{k=1}^{N} d_{k}(t)
$$

After the code multiplexing step, the signal $d(t)$ is transposed to the baseband frequency by an IQ demodulator.

IQ imbalance: This RF impairement means that there is a loss of orthogonality between the I and Q branches, which is due to a gain mismatch $g$ and a phase mismatch $\varphi$ between the two local oscillators [7]. The phase mismatch is caused by a non-ideal layout which means that the lines between mixers are not strictly equal. The gain mismatch is due to a difference of the conversion gain between the I and Q mixers. By taking into account IQ imbalance, the in-phase component $I(t)$ and the in-quadrature component $Q(t)$ are:

$$
\begin{gathered}
I(t)=L P\left[d(t) \cos \left(2 \pi f_{0} t\right)\right], \\
Q(t)=L P\left[d(t) g \sin \left(2 \pi f_{0} t+\varphi\right)\right],
\end{gathered}
$$

where $L P[\bullet]$ refers to an ideal low-pass filter having a bandwidth of $B W / 2(B W$ is bandwidth of $d(t))$.

By replacing $d(t)$ by (11) and (10), we get:

$$
\begin{aligned}
I(t) & =\sum_{k=1}^{N} \frac{1}{2}\left(x_{k}(t)+x_{k}^{*}(t)\right) c_{k}^{p e r}(t), \\
Q(t) & =\sum_{k=1}^{N} g \frac{j}{2}\left(x_{k}^{*}(t) e^{j \varphi}-x_{k}(t) e^{-j \varphi}\right) c_{k}^{p e r}(t) .
\end{aligned}
$$

After demodulation, the signal is defined by its complex envelope:

$$
\hat{x}(t)=I(t)+j Q(t)=\sum_{k=1}^{N} \frac{1}{2}\left[x_{k}(t) K_{1}+x_{k}^{*}(t) K_{2}\right] c_{k}^{p e r}(t),
$$

with $\left\{\begin{array}{l}K_{1}=1+g e^{j \varphi} \\ K_{2}=1-g e^{-j \varphi}\end{array}\right.$.

Digital decoding: So far, the different steps will be implemented in the analog part of the receiver. The decoding step will be performed digitally after sampling.

The pseudo-random sequence modulating the symbols during the spreading step has to be known by the decoder in order to enable the reconstruction of symbols by successive correlations (despreading and integration on the symbol time) between the spreading signal and the same coding sequence:

$$
\begin{aligned}
\hat{x}_{l}[m] & =\frac{1}{T_{s}} \int_{m T_{s}}^{(m+1) T_{s}} \hat{x}(t) c_{l}^{*}(t) d t, \quad m T_{s} \leq t<(m+1) T_{s}, \\
& =\frac{1}{T_{s}} \sum_{k=1}^{N}\left\{x_{k}[m] K_{1}+x_{k}^{*}[m] K_{2}\right\} \int_{0}^{T_{s}} c_{k}(t) c_{l}^{*}(t) d t .
\end{aligned}
$$

Intercorrelation properties influence the performances of a spread spectrum system that operates by correlation between 
signals and codes. Intercorrelation functions of the codes $\gamma_{k, l}(\tau)$ are defined by:

$$
\gamma_{k, l}(\tau)=\frac{1}{T_{s}} \int_{0}^{T_{s}} c_{k}(t-\tau) c_{l}^{*}(t) d t
$$

If the codes are orthogonals, we have:

$$
\gamma_{k, l}(0)=\delta[k-l] .
$$

As a final result, we get:

$$
\begin{aligned}
\hat{x}_{l}[m] & =\sum_{k=1}^{N}\left\{x_{k}[m] K_{1}+x_{k}^{*}[m] K_{2}\right\} \gamma_{k, l}(0), \\
& =x_{l}[m] K_{1}+x_{l}^{*}[m] K_{2} .
\end{aligned}
$$

The digital baseband symbols $\left\{x_{l}[m], l=1 \ldots N, m \in \mathbb{Z}\right\}$ received on each antenna are recovered. When there is no IQ imbalance (i.e. $K_{1}=1$ and $K_{2}=0$ ) an ideal demodulation is possible corresponding to $\hat{x}_{l}[m]=x_{l}[m]$.

The IQ imbalance results depicted in (22) is the same as classical IQ receiver [7].

\section{B. Simulation results}

Fig. 4 gives the normalized BER evolution as a function of the gain and the phase imbalance. The power level conditions leads to a $10^{-3}$ level of BER under ideal IQ mismatch conditions. Simulated performances of the code multiplexing architecture are compared to the performances of a classical homodyne structure. Simulations have been done in the same conditions described in the next section, the SMI processing is not considered in this results.

According to theory, simulation results show that the new code multiplexing architecture has nearly the same sensibility as the classical homodyne architecture.
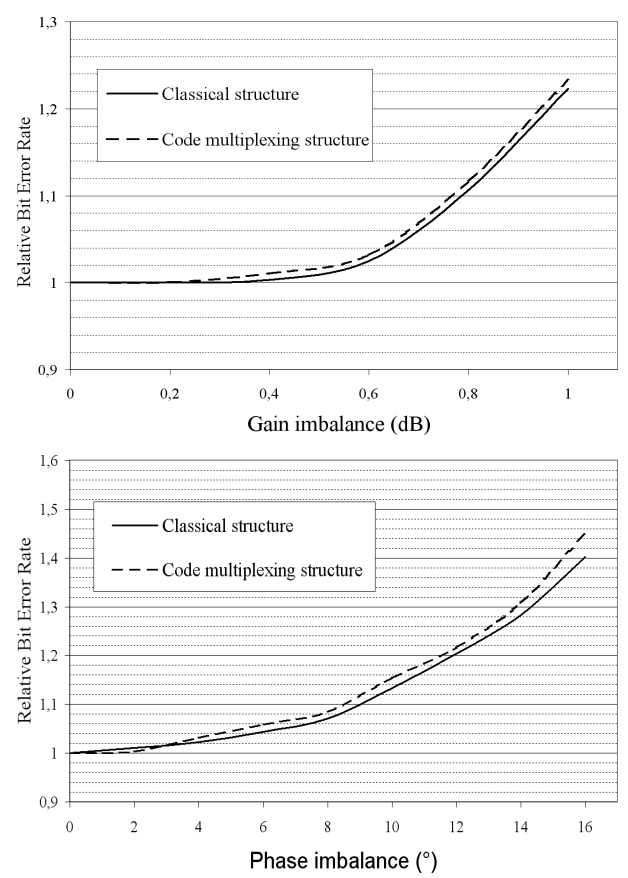

Fig. 4. Relative BER versus gain imbalance and versus phase imbalance.

\section{Simulated MUlti-ANTENNA PERFormanceS}

A global multi-antenna system has been tested by using several BER simulations, it includes the front-end part and the signal processing part. Two complete IEEE 802.11g [2] SIMO transmission systems have been modelled using the ADS software: one using the classical homodyne front-end, the other using the code multiplexing front-end.

\section{A. System description}

The implemented system is described in Fig 3. A two antennas system is tested because experimental validation can be done only with two antennas. Each antenna receives a different version of the $802.11 \mathrm{~g}$ transmitted signal. The channel bandwidth is $20 \mathrm{MHz}$, the RF frequency is $2412 \mathrm{MHz}$ and the datarate is $36 \mathrm{Mbit} / \mathrm{s}$.

We consider a perfect code synchronization. We choose WalshHadamard codes [10] as those used by the UMTS standard. The signal $r_{1}(t)$ from the first antenna is not spread because its associated code $c_{1}(t)$ is only composed of ' 1 '. Thus, the performances are given only for the second antenna which is coded by a non-unitary code $c_{2}(t)$.

\section{B. Simulations results}

In a first time, no IQ imbalance is considered. The influence of SMI processing is shown in Fig. 5 for the two structures. We consider only the transmission quality of the second antenna, but the results are equals for each antenna.

The BER evolutions show that, in an ideal case without imperfections (no multipath channel, no IQ imbalance), a $3 \mathrm{~dB}$ gain is achieved using SMI processing for the two structures. This processing gain is the theoretical results that a two antennas system should reach [3].

The structure using code multiplexing decreases the performances by less than $0.5 \mathrm{~dB}$ at a $B E R=10^{-2}$. This degradation is due to digital aliasing of the noise during the subsampling step after filtering.

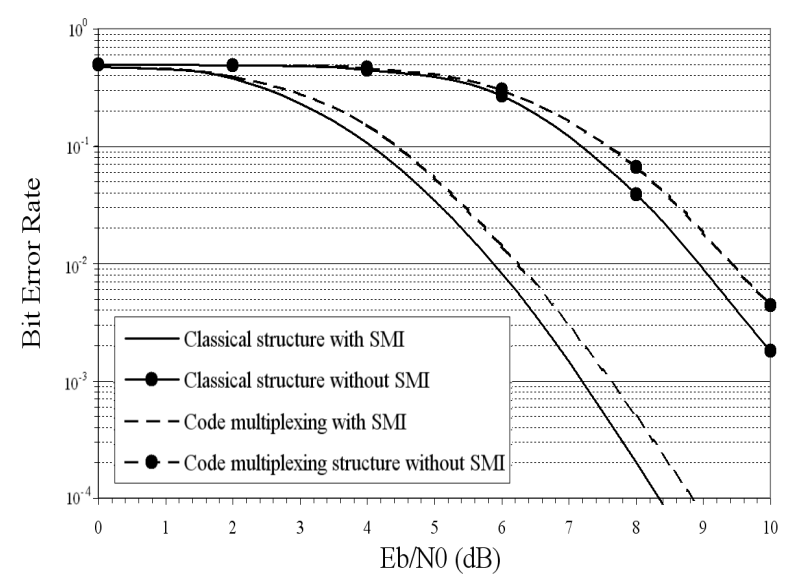

Fig. 5. BER versus $\frac{E b}{N_{0}}$ with or without SMI. 


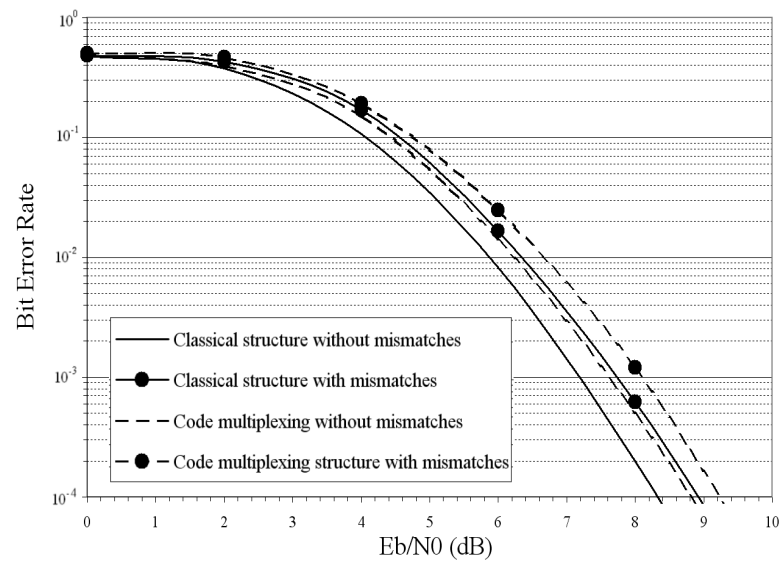

Fig. 6. BER versus $\frac{E b}{N_{0}}-\phi=5^{\circ}$ and $g=1 \mathrm{~dB}$.

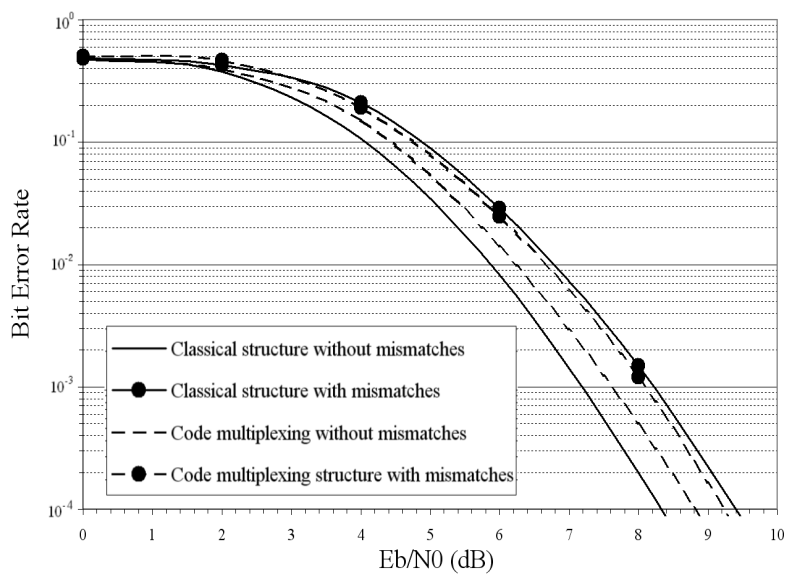

Fig. 7. BER versus $\frac{E b}{N_{0}}-\phi= \pm 5^{\circ}$ and $g= \pm 1 \mathrm{~dB}$.

Fig. 6 and Fig. 7 show the BER evolution versus $\frac{E b}{N_{0}}$ for two different IQ imbalance cases of the classical structure. In a first step, the two IQ demodulators have the same imbalance ( $\phi=5^{\circ}$ and $g=1 \mathrm{~dB}$ ). In a second step, one IQ demodulator has an imbalance set to $\phi=5^{\circ}$ and $g=1 \mathrm{~dB}$, the second one has the opposite value $\left(\phi=-5^{\circ}\right.$ and $\left.g=-1 \mathrm{~dB}\right)$. This second case is more realistic than the first one. The code multiplexing architecture uses only one demodulator which is set to $\phi=5^{\circ}$ and $g=1 \mathrm{~dB}$ for the two cases.

Compared to the performances without mismatches, the IQ imbalance induces a $0.5 \mathrm{~dB}$ loss at a $B E R=10^{-2}$ for the two structures when the mismatches is equal for all IQ demodulators. When the IQ mismatches are different between the two demodulators of the classical structure, a $1 \mathrm{~dB}$ loss is reached instead of $0.5 \mathrm{~dB}$ for the code multiplexing structure. With the realistic assumption of two different imbalances, the two structures have nearly the same performances, the code multiplexing having a lower complexity.

\section{Conclusions}

A novel architecture for an antenna diversity receiver has been introduced. The proposed structure uses orthogonal codes to multiplex the different branches through a single IQ demodulator. The main goal was to reduce the complexity of the analog front-end.

In this paper, we demonstrate an interesting property of this structure concerning its IQ imbalance sensibility: the resulting IQ influence is the same for each baseband contribution whereas the IQ influence depends on each dedicated IQ demodulator for a classical structure. Associated to SMI algorithm, this property allows an IQ sensibility reduction of the multi-antenna system.

The follow-ups of this work are to accurately define the specifications of the analog components to reduce the digital aliasing due to the subsampling step. An extension to multichannel receiver has to be studied as well as its resulting complexity-performance trade-off.

\section{ACKNOWLEDGMENT}

The authors would like to thank Orange Labs which supporte this study.

\section{REFERENCES}

[1] J.H. Winters, "Optimum Combining in Digital Mobile Radio with Cochannel Interference," IEEE Journal on Selected Areas in Communications, vol. 2, no. 4, pp. 528-539, July 1984.

[2] "Wireless MAN Medium Access Control and Physical Specification," IEEE Std 802.11, 1999.

[3] T.K. Sarkar, M.C. Wicks, M. Salazar-Palma, , and R.J. Bonneau, Smart Antennas, Wiley Series in Microwave and Optical Engineering, 2003.

[4] I.J. Gupta, "SMI adaptive antenna arrays for weak interfering signals," IEEE Transactions on Antennas and Propagation, vol. 34, no. 10, pp. 1237-1242, Oct. 1986.

[5] P.F. Morlat, J.C. Nunez Pérez, G. Villemaud, J. Verdier, and J.M. Gorce, "On the Compensation of RF Impairments with Multiple Antennas in SIMO-OFDM Systems," IEEE Vehicular Technology Conference (VTC Fall 2006), September 2006.

[6] M. Gautier and G. Villemaud, "Low complexity antenna diversity front-end: Use of code multiplexing," Proceedings of IEEE Wireless Communication and Networking Conference (WCNC09), April 2009.

[7] S. Traverso, M. Ariaudo, I. Fijalkow, J-L Gautier, and C. Lereau, "Decision Directed Channel Estimation and High I/Q Imbalance Compensation in OFDM Receivers," IEEE Transactions on Communications, Feb. 2008.

[8] H. Tsurumi and Y. Suzuki, "Broadband RF stage architecture for software-defined radio inhandheld terminal applications," IEEE Communications Magazine, vol. 37, no. 2, pp. 90-95, February 1999.

[9] R. Kohno, R. Meidan, and L.B. Milstein, "Spread spectrum access methods for wireless communications," IEEE Communications Magazine, vol. 33, no. 1, pp. 58-67, Janvier 1995.

[10] H. Schulze and C. Lueders, Theory and Applications of OFDM and CDMA: Wideband Wireless Communications, Wiley, 2005. 